\title{
Modeling and Control of a High-thrust Direct-drive Spiral Motor
}

\author{
Yasutaka Fujimoto, Issam A. Smadi, Hiroko Omori, Koichiro Suzuki, and Hiroshi Hamada \\ Department of ECE, Yokohama National University, 79-5 Tokiwadai, Yokohama 240-8501, JAPAN
}

\begin{abstract}
In this paper, modeling and control of spiral motor is proposed. The voltage equation and motion equation of the spiral motor is proposed. Based on this modeling, control system for the spiral motor is proposed. The proposed controller consists of three parts; the first part is PI current controller with back EMF compensation specialized for spiral motor, the second part is disturbance observer based PD controller for linear and gap motion interacting each other, and the third part is zero-power controller for equilibrium fluctuation of gap displacement. It is confirmed that the proposed controller achieves independent linear position and gap control simultaneously.
\end{abstract}

Index Terms-Axial flux machine, Linear actuator, Motion control, Robotics.

\section{INTRODUCTION}

Efficiency and power density of the electric motors are very high compared to other actuators, but its torque is not enough for several applications. Therefore high-ratio gears are combined with electric motors in many applications. Main losses in such actuator systems with the geared servo motors are mechanical transmission loss in the gears, iron loss in the iron core, copper loss in the windings, and switching loss in the power converter. From a control viewpoint, the mechanical loss, i. e., friction loss reduces adaptability, safety, and backdrivability in the motion systems. Various motion mechanisms and controls that recover the backdrivability were reported in the past works[1]-[10].

The authors have proposed a novel helical mechanism of actuator that realizes direct-drive motion without mechanical gears[11]-[13]. The structure and developed prototype are shown in Fig. 1. Permanent magnets are attached on the surfaces of the mover. Slots are provided for winding on the surface of the stator. Three-phase winding in the slots generates flux in the axial direction. Thus, the spiral motor is a helical motion permanent magnet axial flux machine. Due to its large air-gap area, the motor can generate relatively high thrust force.

In this paper, circuit equation and motion equation of the spiral motor are introduced. In order to realize direct-drive motion, we need to keep the air-gap constant. A magnetic levitation control is proposed for this purpose. A variation of the zero power controller is also proposed.

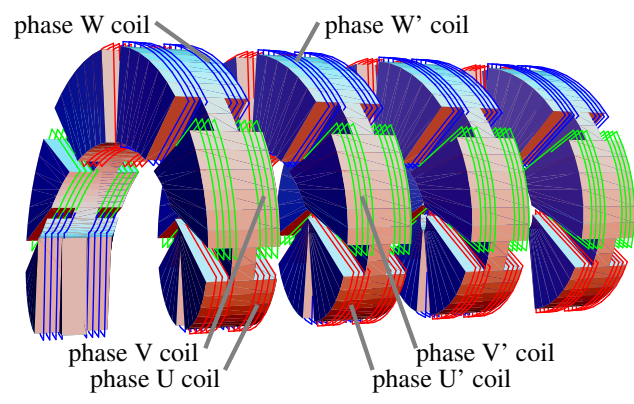

(a) A stator with three-phase windings.

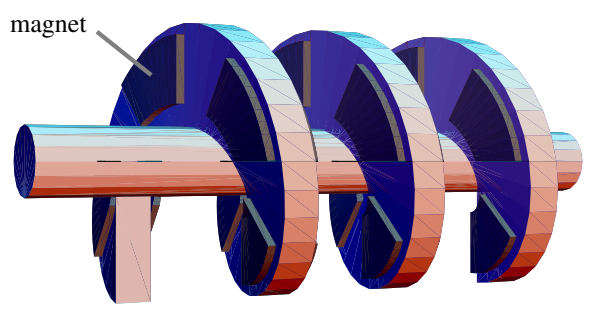

(b) A mover with permanent magnets.

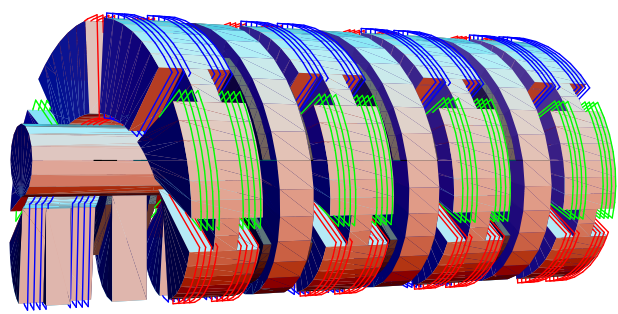

(c) A motor combined with the stator and mover.

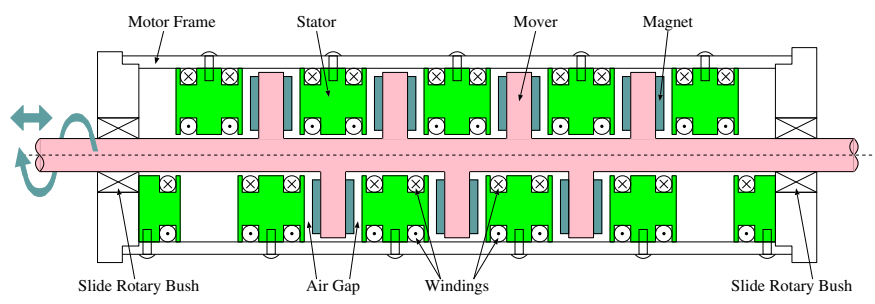

(d) A cross-sectional view.

Fig. 1. A preliminary structure of a spiral motor. 

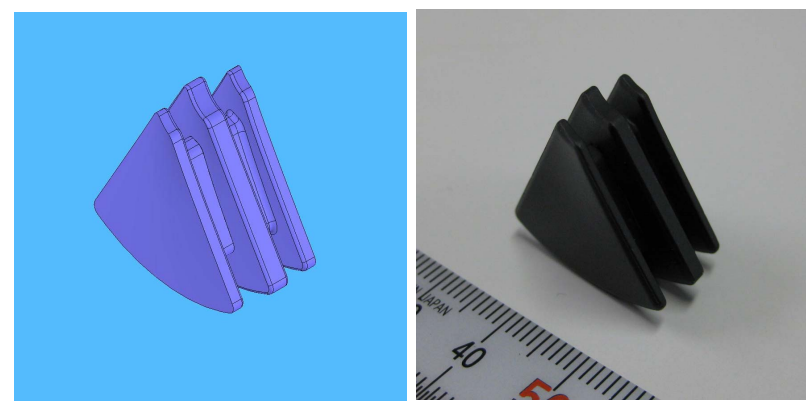

Fig. 2. A helical-shape stator yoke.

\section{MODELING OF SPIRAL MOTOR}

\section{A. Mechanism of Spiral Motor}

Figure 1 (a)-(c) shows a preliminary structure of the spiral motor. Permanent magnets are attached on the surfaces of the mover. Slots are provided for windings on the surface of the stator. The spiral motor is a helical motion axial flux permanent magnet motor. Three-phase windings in the slots generates flux in the axial direction. Due to its large air-gap area, the motor can generate relatively high thrust force.

The cross sectional view of the spiral motor is shown in Fig. 1 (d). The radial load applied to the output shaft is supported by two slide rotary bushes and the thrust load is directly controlled by the electromagnetic force. Thus a magnetic levitation control is required to keep the air-gap constant. For this levitation control, two independent threephase inverters are required.

\section{B. Prototypes}

Four types of prototype of spiral motors have been developed as shown in Table III. A helical-shape stator yoke is made of soft magnetic composite (SMC) as shown in Fig. 2. A helical-shape magnet is made of Nd-Fe-B as shown in Fig. 3. Internal structure of the stator is shown in Fig. 4. The NdFe-B magnets are attached on the mover yoke which is made of silicon steel, as shown in Fig. 5. Precise helical shapes of the stator and mover enable uniform short length of air-gap and avoid concentration of stress when the mover touches down to the stator.

Exterior of the spiral motor is shown in Fig. 6. A rotary encoder and linear encoder attached at the mover measure the linear position $x$ and rotation angle $\theta$ for control. Displacement of the air gap $x_{g}$ is computed by using these measurement as follows.

$$
x_{g}=x-\frac{\ell_{p}}{2 \pi} \theta
$$

\section{Permeance Model}

In order to derive analytical voltage equation, and thrustforce and torque equations, a permeance model of the motor for 360 degree electric angular displacement in polar coordinates is presented.

Figure 7 shows the polar coordinates expression of the motor. From this model, a simple magnetic circuit as shown in Fig. 8 is obtained. For simplicity magnetic resistance of
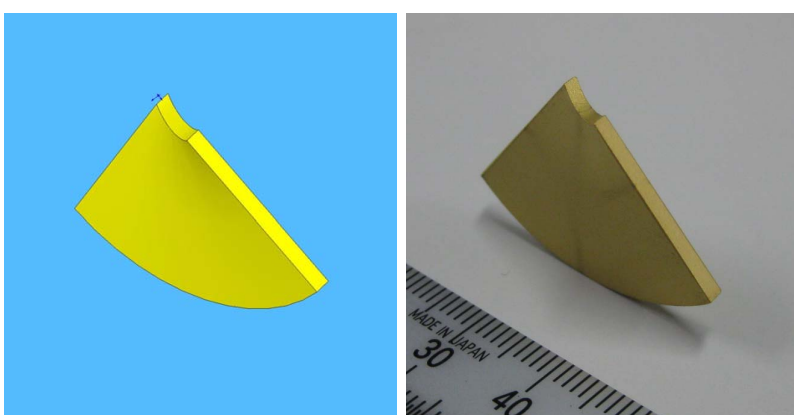

Fig. 3. A helical-shape Nd-Fe-B magnet.

TABLE I

SPECIFICATIONS OF SPIRAL MOTORS.

\begin{tabular}{lcccc}
\hline & $\# 1$ & $\# 2$ & $\# 3$ & $\# 4$ \\
\hline Number of stator layers & 6 & 8.5 & 12 & 12 \\
Number of mover layers & 2 & 4 & 7 & 5 \\
Length of flame*[mm] & 132 & 182 & 252 & 252 \\
Diameter of flame*[mm] & 55 & 55 & 55 & 55 \\
Stroke[mm] & 60 & 70 & 80 & 120 \\
Lead length of screw[mm] & 20 & 20 & 20 & 20 \\
Nominal length of air gap[mm] & 1 & 1 & 1 & 1 \\
\hline
\end{tabular}

*excluding projecting parts
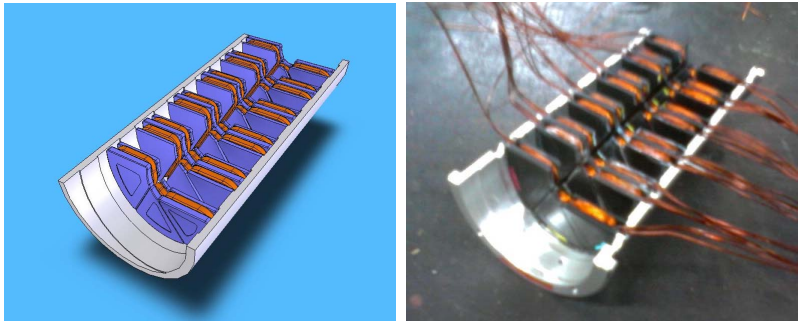

Fig. 4. Internal structure of a stator. (model \#1)

the iron core is ignored. $R_{g}=3 p\left(\ell_{g}-x_{g}\right) / S \mu_{0}$ is magnetic resistance of forward side air gap for an area of each phase windings, where $S$ is a gap area of the cross section of the cylinder. $R_{g}^{\prime}=3 p\left(\ell_{g}+x_{g}\right) / S \mu_{0}$ is magnetic resistance of backward side air gap. $R_{m}=3 p \ell_{m} / S \mu_{m}$ is magnetic resistance of a permanent magnet. $c(\cdot) I_{f}$ is a spatial function of magnetomotive force of the field magnet by the permanent magnet and approximated by a cosine function as $c(\theta)=$ $k \cos (\theta)$ where $k=\frac{6 \sqrt{3}}{\pi^{2}} \sin (p \alpha / 2)$ is the fundamental component of $c(\theta) . \quad I_{f}=B_{r} \ell_{m} / \mu_{m}$ is an equivalent magnetization current, i. e., magnetomotive force of the permanent magnet. These parameters are shown in Table II. For simplicity, we assume that the permeability of the permanent magnet $\mu_{m}$ is equivalent to $\mu_{0}$. Also the edge effect is ignored.

In the part (A) of the magnetic circuit, interlinkage flux $\Phi=\left[\Phi_{u}, \Phi_{v}, \Phi_{w}, \Phi_{f}\right]^{T}$ for each current is obtained as $\Phi=$ $L I$ where $I=\left[I_{u}, I_{v}, I_{w}, I_{f}\right]^{T}$ is current vector and $L$ is an inductance matrix:

$$
L=P\left[\begin{array}{cccc}
n^{2} & -\frac{1}{2} n^{2} & -\frac{1}{2} n^{2} & \frac{3}{2} n k c_{0} \\
-\frac{1}{2} n^{2} & n^{2} & -\frac{1}{3} n^{2} & \frac{3}{2} n k c_{1} \\
-\frac{1}{2} n^{2} & -\frac{1}{2} n^{2} & n^{2} & \frac{3}{2} n k c_{2} \\
\frac{3}{2} n k c_{0} & \frac{3}{2} n k c_{1} & \frac{3}{2} n k c_{2} & \left(\frac{3 k}{2}\right)^{2}
\end{array}\right]
$$



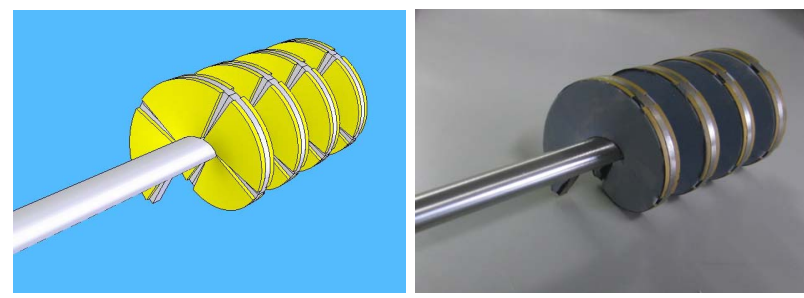

Fig. 5. A mover. (model \#2)

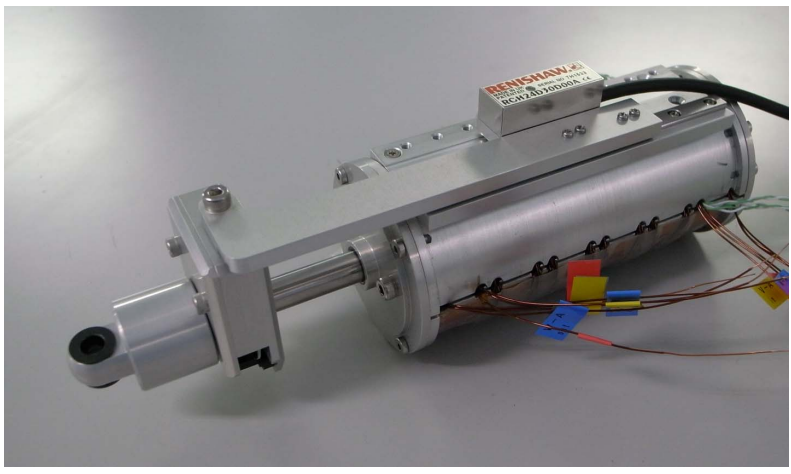

Fig. 6. Exterior of a spiral motor. (model \#1)
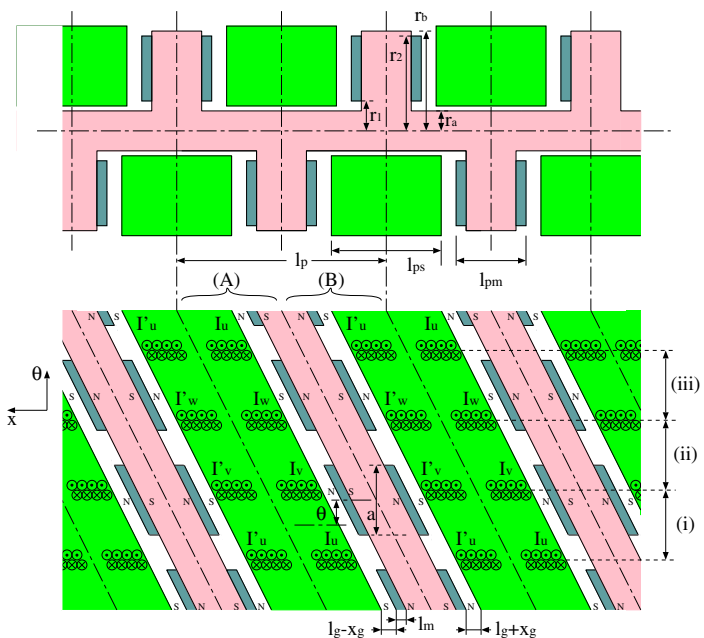

Fig. 7. A spiral motor in polar coordinates.

where $P=\frac{2}{3\left(R_{g}+R_{m}\right)}$ is permeance for one-phase current and $c_{i}=\cos \left(p \theta-\frac{2 i \pi}{3}\right)$.

In general, a salient-mover machine has a mutual inductance between $n_{i}$-turns windings at position $\theta_{i}$ and $n_{j}$-turns windings at position $\theta_{j}$ represented by

$$
\begin{aligned}
L_{i j}= & P_{d} n_{i} n_{j} \cos \left(\theta_{i}-p \theta\right) \cos \left(\theta_{j}-p \theta\right) \\
& +P_{q} n_{i} n_{j} \sin \left(\theta_{i}-p \theta\right) \sin \left(\theta_{j}-p \theta\right)
\end{aligned}
$$

where $P_{d} \propto 1 /\left(\ell-x_{g}\right)$ is $\mathrm{d}$-axis permeance and $P_{q} \propto$ $1 /\left(\ell-x_{g}\right)$ is $\mathrm{q}$-axis permeance. $\ell=\ell_{g}+\ell_{m}$ is a nominal gap between the mover iron and the stator. Instead of (2), we

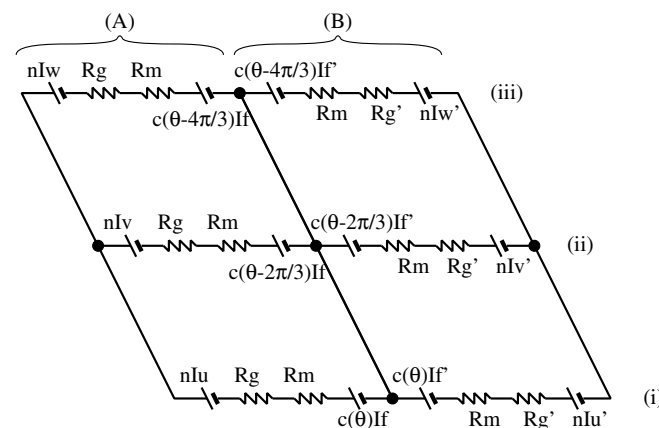

Fig. 8. A magnetic circuit.

TABLE II

\begin{tabular}{|c|c|}
\hline$\alpha$ & angle of a sector-type permanent magnet \\
\hline$B_{r}$ & residual flux density of a permanent magnet \\
\hline$f$ & thrust force of the mover \\
\hline$I_{f}$ & magnetomotive force of a field magnet \\
\hline$I_{i}$ & $i$-axis current on forward side windings, $i \in\{d, q\}$ \\
\hline$I_{i}^{\prime}$ & $i$-axis current on backward side windings, $i \in\{d, q\}$ \\
\hline$J$ & moment of inertia of the mover around the axis \\
\hline$k$ & a fundamental Fourier component of $c(\theta)$ \\
\hline$\ell$ & a nominal gap between the mover iron and the stator \\
\hline$\ell_{g}$ & nominal length of air gap \\
\hline$\ell_{m}$ & thickness of magnet \\
\hline$\ell_{p}$ & lead length of screw \\
\hline$\mu_{0}$ & the permeability in vacuum \\
\hline$\mu_{m}$ & permeability of a permanent magnet \\
\hline$M$ & mass of the mover \\
\hline$n$ & ampere-turn \\
\hline$p$ & $\begin{array}{l}\text { number of pole pairs per } 360 \text { degree mechanical } \\
\text { angular displacement }\end{array}$ \\
\hline$q$ & number of layers \\
\hline$\theta$ & mechanical rotational angle of the mover \\
\hline$S$ & $\begin{array}{l}\text { a gap area per } 360 \text { degree mechanical angle } \\
\text { displacement }\end{array}$ \\
\hline$\tau$ & torque of the mover \\
\hline$x$ & a linear position of the mover \\
\hline$x_{g}$ & displacement of air gap \\
\hline
\end{tabular}

NOMENCLATURE

adopt a inductance matrix as follows

$$
L=\left[\begin{array}{llll}
L_{00} & L_{01} & L_{02} & L_{03} \\
L_{10} & L_{11} & L_{12} & L_{13} \\
L_{20} & L_{21} & L_{22} & L_{23} \\
L_{30} & L_{31} & L_{32} & L_{33}
\end{array}\right]
$$

where $n_{0}=n_{1}=n_{2}=n, n_{3}=n_{f}=3 k / 2, \theta_{0}=0, \theta_{1}=$ $2 \pi / 3, \theta_{2}=4 \pi / 3$, and $\theta_{3}=p \theta$. The model (4) is equivalent to (2) if the machine is not salient, i. e., $P_{d}=P_{q}=P$ holds.

The voltage equation, thrust-force equation, and torque equation of the spiral motor are derived by using the inductance matrix (4) as follows.

$$
\begin{aligned}
V & =R I+L \frac{d I}{d t}+\dot{\theta} \frac{\partial L}{\partial \theta} I+\dot{x} \frac{\partial L}{\partial x} I \\
f & =\frac{1}{2} I^{T} \frac{\partial L}{\partial x} I \\
\tau & =\frac{1}{2} I^{T} \frac{\partial L}{\partial \theta} I
\end{aligned}
$$

where $V=\left[V_{u}, V_{v}, V_{w}, V_{f}\right]^{T}$ is voltage of each windings, 
$R=\operatorname{diag}\left(R_{s}, R_{s}, R_{s}, R_{f}\right)$ is resistance of windings, and $f$ and $\tau$ are thrust-force and torque of the mover, respectively.

\section{Voltage Equation on dq-axis}

In order to apply field oriented control to the spiral motor, dq-axis model of the spiral motor is derived as follows. Let the transformation matrix $C$ be

$$
C=\left[\begin{array}{cccc}
\sqrt{\frac{2}{3}} c_{0} & \sqrt{\frac{2}{3}} c_{1} & \sqrt{\frac{2}{3}} c_{2} & 0 \\
-\sqrt{\frac{2}{3}} s_{0} & -\sqrt{\frac{2}{3}} s_{1} & -\sqrt{\frac{2}{3}} s_{2} & 0 \\
\frac{1}{\sqrt{3}} & \frac{1}{\sqrt{3}} & \frac{1}{\sqrt{3}} & 0 \\
0 & 0 & 0 & 1
\end{array}\right]
$$

where $c_{i}=\cos \left(p \theta-\frac{2 i \pi}{3}\right), s_{i}=\sin \left(p \theta-\frac{2 i \pi}{3}\right)$. Then the dq-axis current and voltage are represented by $I_{d q}=C I$ and $V_{d q}=$ $C V$ where $I_{d q}=\left[I_{d}, I_{q}, I_{0}, I_{f}\right]^{T}, V_{d q}=\left[V_{d}, V_{q}, V_{0}, V_{f}\right]^{T}$. And $I_{d}, I_{q}, I_{0}$ are d-axis, q-axis, and zero-phase current, $V_{d}$, $V_{q}, V_{0}$ are d-axis, q-axis, and zero-phase voltage, respectively.

The voltage equation on dq-axis is

$$
\begin{aligned}
V_{d q}= & C R C^{T} I_{d q}+C L C^{T} \dot{I}_{d q}+\dot{\theta} C \frac{\partial L}{\partial \theta} C^{T} I_{d q} \\
& +\dot{\theta} C L \frac{\partial C^{T}}{\partial \theta} I_{d q}+\dot{x} C \frac{\partial L}{\partial x} C^{T} I_{d q}
\end{aligned}
$$

In (9), note that

$$
\begin{aligned}
\frac{\partial L}{\partial \theta} & =\left.\frac{\partial L\left(x_{g}, \theta\right)}{\partial \theta}\right|_{x_{g}=x-\frac{\ell_{p}}{2 \pi} \theta} \\
& =\frac{\partial L\left(x_{g}, \theta\right)}{\partial \theta}-\frac{\ell_{p}}{2 \pi} \frac{\partial L\left(x_{g}, \theta\right)}{\partial x_{g}}
\end{aligned}
$$

Then, the voltage equation (9) is expanded as follows.

$$
\begin{aligned}
V_{d}= & R_{s} I_{d}+L_{d} \dot{I}_{d}-p \dot{\theta} L_{q} I_{q}+L_{d f} \dot{I}_{f} \\
& +\frac{\dot{x}_{g}}{\ell-x_{g}}\left(L_{d} I_{d}+L_{d f} I_{f}\right) \\
V_{q}= & R_{s} I_{q}+L_{q} \dot{I}_{q}+p \dot{\theta}\left(L_{d} I_{d}+L_{d f} I_{f}\right) \\
& +\frac{\dot{x}_{g}}{\ell-x_{g}} L_{q} I_{q} \\
V_{0}= & R_{s} I_{0} \\
V_{f}= & L_{f} \dot{I}_{f}+L_{d f} \dot{I}_{d}+\frac{\dot{x}_{g}}{\ell-x_{g}}\left(L_{d} I_{f}+L_{d f} I_{d}\right)
\end{aligned}
$$

where $L_{d}=\frac{3}{2} n^{2} P_{d} \propto 1 /\left(\ell-x_{g}\right)$ and $L_{q}=\frac{3}{2} n^{2} P_{q} \propto 1 /(\ell-$ $\left.x_{g}\right)$ are $\mathrm{d}$-axis and q-axis inductances, respectively. $L_{d f}=$ $\sqrt{\frac{3}{2}} n n_{f} P_{d} \propto 1 /\left(\ell-x_{g}\right)$ is equivalent mutual inductance between $\mathrm{d}$-axis stator windings and field magnet windings. $L_{f}=n_{f}^{2} P_{d} \propto 1 /\left(\ell-x_{g}\right)$ is equivalent self-inductance of field magnet windings.

Finally, following dq-axis voltage equation is obtained by taking first two equations (11), (12) and substituting $\dot{I}_{f}=0$.

$$
\begin{aligned}
{\left[\begin{array}{c}
V_{d} \\
V_{q}
\end{array}\right]=} & {\left[\begin{array}{cc}
R_{s}+L_{d}\left(\frac{d}{d t}\right) & 0 \\
0 & R_{s}+L_{q}\left(\frac{d}{d t}\right)
\end{array}\right]\left[\begin{array}{c}
I_{d} \\
I_{q}
\end{array}\right]+\Psi_{f}\left[\begin{array}{c}
\frac{1}{\ell-x_{g}} \dot{x}_{g} \\
p \dot{\theta}
\end{array}\right] } \\
& +\frac{\dot{x}_{g}}{\ell-x_{g}}\left[\begin{array}{cc}
L_{d} & 0 \\
0 & L_{q}
\end{array}\right]\left[\begin{array}{c}
I_{d} \\
I_{q}
\end{array}\right]+p \dot{\theta}\left[\begin{array}{cc}
0 & -L_{q} \\
L_{d} & 0
\end{array}\right]\left[\begin{array}{c}
I_{d} \\
I_{q}
\end{array}\right]
\end{aligned}
$$

where $\Psi_{f}=L_{d f} I_{f}$ corresponds to field flux by the permanent magnet. Fig. 9 shows block diagram of this dq-axis circuit model.

As the same manner, voltage equation of part (B) in Fig. 7 and 8 is obtained as follows.

$$
\begin{aligned}
{\left[\begin{array}{c}
V_{d}^{\prime} \\
V_{q}^{\prime}
\end{array}\right]=} & {\left[\begin{array}{cc}
R_{s}+L_{d}^{\prime}\left(\frac{d}{d t}\right) & 0 \\
0 & R_{s}+L_{q}^{\prime}\left(\frac{d}{d t}\right)
\end{array}\right]\left[\begin{array}{c}
I_{d}^{\prime} \\
I_{q}^{\prime}
\end{array}\right]+\Psi_{f}^{\prime}\left[\begin{array}{c}
-\frac{1}{\ell+x_{g}} \dot{x}_{g} \\
p \dot{\theta}
\end{array}\right] } \\
& -\frac{\dot{x}_{g}}{\ell+x_{g}}\left[\begin{array}{cc}
L_{d}^{\prime} & 0 \\
0 & L_{q}^{\prime}
\end{array}\right]\left[\begin{array}{c}
I_{d}^{\prime} \\
I_{q}^{\prime}
\end{array}\right]+p \dot{\theta}\left[\begin{array}{cc}
0 & -L_{q}^{\prime} \\
L_{d}^{\prime} & 0
\end{array}\right]\left[\begin{array}{c}
I_{d}^{\prime} \\
I_{q}^{\prime}
\end{array}\right]
\end{aligned}
$$

where $L_{d}^{\prime}=\frac{3}{2} n^{2} P_{d}^{\prime} \propto 1 /\left(\ell+x_{g}\right)$ and $L_{q}^{\prime}=\frac{3}{2} n^{2} P_{q}^{\prime} \propto$ $1 /\left(\ell+x_{g}\right)$ are $\mathrm{d}$-axis and q-axis inductances in part (B), respectively. $\Psi_{f}^{\prime}=L_{d f}^{\prime} I_{f}^{\prime}$ corresponds to field flux by the permanent magnet in part (B), where $L_{d f}^{\prime}=\sqrt{\frac{3}{2}} n n_{f} P_{d}^{\prime} \propto$ $1 /\left(\ell+x_{g}\right)$.

Two independent three-phase inverters are required for driving the spiral motor. One inverter controls the circuit (15) and the other controls the circuit (16).

\section{E. Thrust-force/Torque Equation}

Thrust-force equation (6) and torque equation (7) are rewritten by using $I_{d q}$ as follows.

$$
\begin{aligned}
f & =\frac{1}{2} I_{d q}^{T} C \frac{\partial L}{\partial x} C^{T} I_{d q} \\
& =\frac{1}{\ell-x_{g}}\left(\Psi_{f} I_{d}+\frac{L_{d} I_{d}^{2}+L_{q} I_{q}^{2}+L_{f} I_{f}^{2}}{2}\right) \\
\tau & =\frac{1}{2} I_{d q}^{T} C \frac{\partial L}{\partial \theta} C^{T} I_{d q} \\
& =p\left(\Psi_{f} I_{q}+\left(L_{d}-L_{q}\right) I_{d} I_{q}\right) \\
- & \frac{\ell_{p}}{2 \pi} \frac{1}{\ell-x_{g}}\left(\Psi_{f} I_{d}+\frac{L_{d} I_{d}^{2}+L_{q} I_{q}^{2}+L_{f} I_{f}^{2}}{2}\right)
\end{aligned}
$$

As the same manner, thrust-force equation and torque equation in part (B) are obtained as follows.

$$
\begin{aligned}
f^{\prime} & =-\frac{1}{\ell+x_{g}}\left(\Psi_{f}^{\prime} I_{d}^{\prime}+\frac{L_{d}^{\prime} I_{d}^{\prime 2}+L_{q}^{\prime} I_{q}^{\prime 2}+L_{f}^{\prime} I_{f}^{\prime 2}}{2}\right) \\
\tau^{\prime} & =p\left(\Psi_{f}^{\prime} I_{q}^{\prime}+\left(L_{d}^{\prime}-L_{q}^{\prime}\right) I_{d}^{\prime} I_{q}^{\prime}\right) \\
+ & \frac{\ell_{p}}{2 \pi} \frac{1}{\ell+x_{g}}\left(\Psi_{f}^{\prime} I_{d}^{\prime}+\frac{L_{d}^{\prime} I_{d}^{\prime 2}+L_{q}^{\prime} I_{q}^{\prime 2}+L_{f}^{\prime} I_{f}^{\prime 2}}{2}\right)
\end{aligned}
$$

Finally, total thrust-force and torque per 360 degree electrical angle are obtained as $f_{\text {total }}=f+f^{\prime}$ and $\tau_{\text {total }}=\tau+\tau^{\prime}$. Hense, the equation of motion is obtained as follows.

$$
\begin{aligned}
M \ddot{x} & =p q\left(f+f^{\prime}\right)-D \dot{x}-d \\
J \ddot{\theta} & =p q\left(\tau+\tau^{\prime}\right)-D_{\tau} \dot{\theta}-d_{\tau}
\end{aligned}
$$

where $M, J$ are mass and inertia of the mover, respectively. $D, D_{\tau}$ are friction coefficient for linear and rotaty motion, respectively. $d, d_{\tau}$ are disturbance force and torque applied to the mover, respectively. 


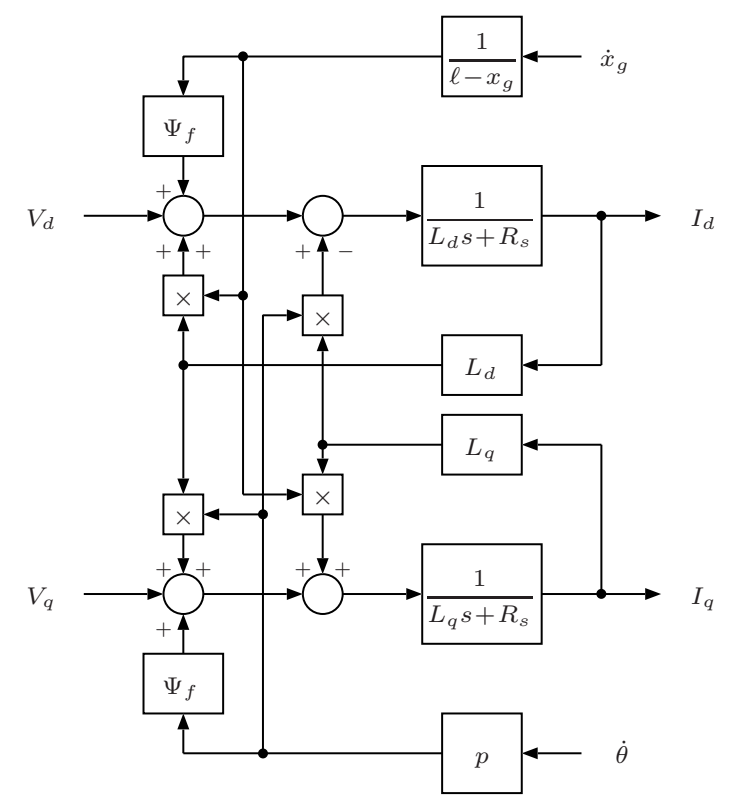

Fig. 9. dq-axis circuit model of the spiral motor in part A.

\section{F. Simplified Thrust-Force/Torque Model}

For control design, a simplified model is obtained by linearlizing (18), (20), (21), and (22) around $x_{g}=0$.

$$
\begin{aligned}
f_{\text {total }} \simeq & \frac{2 L_{f 0} I_{f}^{2}}{\ell^{2}} x_{g}+\frac{\Psi_{f 0}}{\ell}\left(I_{d}-I_{d}^{\prime}\right) \\
& +\frac{L_{d 0}}{2 \ell}\left(I_{d}^{2}-I_{d}^{\prime 2}\right)+\frac{L_{q 0}}{2 \ell}\left(I_{q}^{2}-I_{q}^{\prime 2}\right) \\
\tau_{\text {total }} \simeq & p\left(\Psi_{f 0}\left(I_{q}+I_{q}^{\prime}\right)+\left(L_{d 0}-L_{q 0}\right)\left(I_{d} I_{q}+I_{d}^{\prime} I_{q}^{\prime}\right)\right) \\
& -\frac{\ell_{p}}{2 \pi}\left(\frac{2 L_{f 0} I_{f}^{2}}{\ell^{2}} x_{g}+\frac{\Psi_{f 0}}{\ell}\left(I_{d}-I_{d}^{\prime}\right)\right. \\
& \left.+\frac{L_{d 0}}{2 \ell}\left(I_{d}^{2}-I_{d}^{\prime 2}\right)+\frac{L_{q 0}}{2 \ell}\left(I_{q}^{2}-I_{q}^{\prime 2}\right)\right)
\end{aligned}
$$

where $L_{d 0}=\left.L_{d}\right|_{x_{g}=0}=\left.L_{d}^{\prime}\right|_{x_{g}=0}, L_{q 0}=\left.L_{q}\right|_{x_{g}=0}=\left.L_{q}^{\prime}\right|_{x_{g}=0}$, $\Psi_{f 0}=\left.\Psi_{f}\right|_{x_{g}=0}=\left.\Psi_{f}^{\prime}\right|_{x_{g}=0}$, and $L_{f 0}=\left.L_{f}\right|_{x_{g}=0}=\left.L_{f}^{\prime}\right|_{x_{g}=0}$.

From (25) and (26), linear motion is mainly controlled by d-axis current and rotational motion is mainly controlled by q-axis current. The first term in (25) corresponds to a unstable drift force which is a positive feedback in terms of gap displacement.

When the field oriented control is applied to (15) and (16) and their current hold conditions $I_{d}=-I_{d}^{\prime}$ and $I_{q}=I_{q}^{\prime}$ by the current control, then we have

$$
\begin{aligned}
& f_{\text {total }} \simeq K_{g} x_{g}+K_{f} I_{d} \\
& \tau_{\text {total }} \simeq K_{\tau} I_{q}-\frac{\ell_{p}}{2 \pi}\left(K_{g} x_{g}+K_{f} I_{d}\right)
\end{aligned}
$$

where $K_{g}=\frac{2 L_{f 0} I_{f}^{2}}{\ell^{2}}, K_{f}=\frac{2 \Psi_{f 0}}{\ell}$, and $K_{\tau}=2 p \Psi_{f 0}$. From (23), (24), (27), and (28), it turns out that the motion system has a spring whose coefficient is negative. Figure 10 shows the block diagram of the simplified model of the motion system.

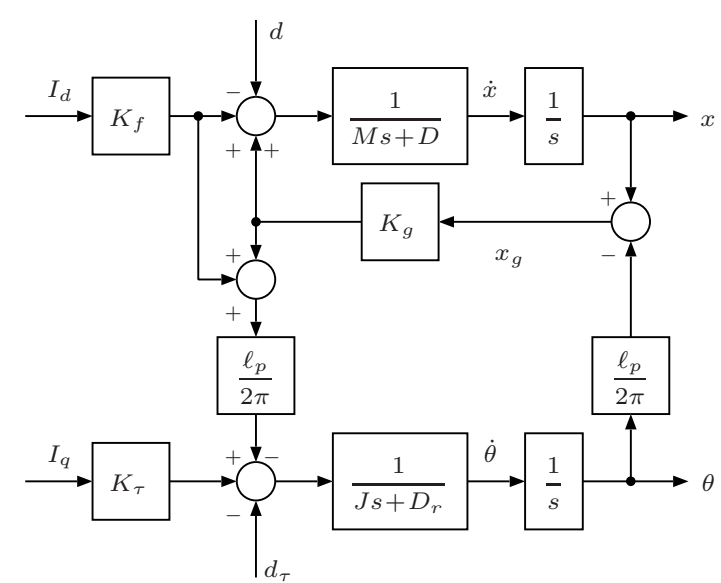

Fig. 10. Simplified motion model of the spiral motor.

\section{Control of the SPIRAL Motor}

\section{A. Current Controller}

From (15) and (16), current controller is designed as follows.

$$
\begin{aligned}
V_{d}^{\text {ref }} & =\left(K_{p d}+\frac{K_{i d}}{s}\right)\left(I_{d}^{\text {ref }}-I_{d}\right)+\hat{E}_{d} \\
V_{q}^{\text {ref }} & =\left(K_{p q}+\frac{K_{i q}}{s}\right)\left(I_{q}^{\text {ref }}-I_{q}\right)+\hat{E}_{q} \\
V_{d}^{\text {ref }} & =\left(K_{p d}+\frac{K_{i d}}{s}\right)\left(-I_{d}^{\text {ref }}-I_{d}^{\prime}\right)+\hat{E}_{d}^{\prime} \\
V_{q}^{\text {'ref }} & =\left(K_{p q}+\frac{K_{i q}}{s}\right)\left(I_{q}^{\text {ref }}-I_{q}^{\prime}\right)+\hat{E}_{q}^{\prime}
\end{aligned}
$$

where the controller gains are chosen so that $K_{p d}=\omega_{i d} L_{d 0}$, $K_{p q}=\omega_{i q} L_{q 0}, K_{i d}=\omega_{i d} R_{s}$, and $K_{i q}=\omega_{i q} R_{s}$. Also $\hat{E}_{d}$, $\hat{E}_{q}, \hat{E}_{d}^{\prime}$, and $\hat{E}_{q}^{\prime}$ are back EMF compensation given by

$$
\begin{aligned}
\hat{E}_{d} & =\frac{\dot{x}_{g}}{\ell}\left(\Psi_{f 0}+L_{d 0} I_{d}\right)-p \dot{\theta} L_{q 0} I_{q} \\
\hat{E}_{q} & =p \dot{\theta}\left(\Psi_{f 0}+L_{d 0} I_{d}\right)+\frac{\dot{x}_{g}}{\ell} L_{q 0} I_{q} \\
\hat{E}_{d}^{\prime} & =-\frac{\dot{x}_{g}}{\ell}\left(\Psi_{f 0}+L_{d 0} I_{d}^{\prime}\right)-p \dot{\theta} L_{q 0} I_{q}^{\prime} \\
\hat{E}_{q}^{\prime} & =p \dot{\theta}\left(\Psi_{f 0}+L_{d 0} I_{d}^{\prime}\right)-\frac{\dot{x}_{g}}{\ell} L_{q 0} I_{q}^{\prime} .
\end{aligned}
$$

\section{B. Position/Gap Controller with Disturbance Observer}

From (27) and (28), current references are obtained as follows.

$$
\begin{aligned}
I_{d}^{r e f} & =\frac{1}{K_{f}}\left(f^{r e f}-K_{g} x_{g}\right) \\
I_{q}^{r e f} & =\frac{1}{K_{\tau}}\left(\frac{\ell_{p}}{2 \pi} f^{r e f}+\tau^{r e f}\right)
\end{aligned}
$$

where $f^{r e f}$ and $\tau^{r e f}$ correspond to thrust-force and torque references. The modeling error and disturbance are compensated by using disturbance observer[2] as follows.

$$
\begin{aligned}
& f^{r e f}=M_{n} \ddot{x}^{r e f}+\hat{d} \\
& \tau^{r e f}=J_{n} \ddot{\theta}^{r e f}+\hat{d}_{\tau}
\end{aligned}
$$


where the terms $\hat{d}$ and $\hat{d}_{\tau}$ are estimated disturbandes.

$$
\begin{aligned}
\hat{d} & =\frac{\omega_{d}}{s+\omega_{d}}\left(f^{r e f}-M_{n} s \dot{x}\right) \\
\hat{d}_{\tau} & =\frac{\omega_{d}}{s+\omega_{d}}\left(\tau^{r e f}-J_{n} s \dot{\theta}\right)
\end{aligned}
$$

The acceleration references in (39) and (40) are computed by trajectory tracking feedback controller for position $x$ and regulator for gap displacement $x_{g}$ as follows.

$$
\begin{aligned}
\ddot{x}^{r e f} & =K_{p x}\left(x^{c m d}-x\right)+K_{d x}\left(\dot{x}^{c m d}-\dot{x}\right)+\ddot{x}^{c m d}(43) \\
\ddot{x}_{g}^{r e f} & =K_{p g}\left(x_{g}^{c m d}-x_{g}\right)+K_{d g}\left(\dot{x}_{g}^{c m d}-\dot{x}_{g}\right) \\
\ddot{\theta}^{r e f} & =\frac{2 \pi}{\ell_{p}}\left(\ddot{x}^{r e f}-\ddot{x}_{g}^{r e f}\right)
\end{aligned}
$$

where $x^{c m d}$ represents position command of the mover, $x_{g}^{c m d}$ represents gap displacement command, respectively. Ideally, the unstable equilibrium point corresponds to being the gap displacement $x_{g}=0$. However, there is a offset of the equilibrium point according to the manufacturing accuracy of the parts of the stator and mover. Thrust load also affects the offset. In such a case, there remains constant current in order to achieve $x_{g}=0$, which causes copper loss in the windings. $x_{g}^{c m d}$ should be set to be the equilibrium point by using the zero power controller.

\section{Zero Power Controller}

Zero-power control[14] was proposed for electromagnetic suspension systems using permanent magnets, which achieves automatic gap adjustment so that the input current converges to zero. In this paper, we propose a variation of zero-power controller suitable for our control system.

The equilibrium gap displacement $x_{g}^{c m d}$ in (44) is obtained by integrating d-axis current reference $I_{d}^{r e f}$ as follows. $x_{g}^{c m d}$ approaches to the equilibrium point and finally $I_{d}=0$ is achieved if $x_{g}^{c m d}$ corresponds to the equilibrium.

$$
\begin{aligned}
& x_{g}^{c m d}=\frac{K_{z}}{s} I_{d}^{r e f} \\
& \dot{x}_{g}^{c m d}=K_{z} I_{d}^{r e f}
\end{aligned}
$$

where $K_{z}$ represents a controller gain.

\section{Overall Control System}

Finally, the overall control system of the spiral motor is described as shown in Fig. 11.

\section{Simulation}

\section{A. Plant and Control Parameters}

Table III shows plant and control parameters. The electrical plant parameters in this table are for 360 degree electrical angle.
TABLE III

PLANT AND CONTROL PARAMETERS

\begin{tabular}{lll}
\hline$p$ & number of pole pairs per 360 degree & 2 \\
$q$ & number of layers & 2 \\
$\ell_{g}$ & nominal length of air gap & $1[\mathrm{~mm}]$ \\
$\ell_{m}$ & thickness of magnet & $2[\mathrm{~mm}]$ \\
$B_{r}$ & residual flux density of magnet & $1.2[\mathrm{~T}]$ \\
$n$ & ampere-turn & 50 \\
$R_{s}$ & registance of windings & $0.374[\Omega]$ \\
$L_{d 0}$ & nominal d-axis inductance & $0.329[\mathrm{mH}]$ \\
$L_{q 0}$ & nominal q-axis inductance & $0.329[\mathrm{mH}]$ \\
$\Psi_{f 0}$ & nominal field flux & $0.0195[\mathrm{~Wb}]$ \\
$K_{f}$ & thrust-force constant & $13.0[\mathrm{~N} / \mathrm{A}]$ \\
$K_{\tau}$ & torque constant & $0.0781[\mathrm{~N} . \mathrm{m} / \mathrm{A}]$ \\
$J$ & moment of inertia of the mover & $715\left[\mathrm{~g} \mathrm{~cm}{ }^{2}\right]$ \\
$M$ & mass of the mover & $0.229[\mathrm{~kg}]$ \\
$K_{g}$ & equivalent spring coefficient & $25800[\mathrm{~N} / \mathrm{m}]$ \\
\hline$K_{p d}, K_{p q}$ & propotional gain of current controller & 1.64 \\
$K_{i d}, K_{i q}$ & integral gain of current controller & 1870 \\
$\omega_{i d}, \omega_{i q}$ & bandwidth of current control system & $5000[\mathrm{rad} / \mathrm{sec}]$ \\
$\omega_{d}$ & bandwidth of disturbance observer & $500[\mathrm{rad} / \mathrm{s}]$ \\
$K_{p x}$ & propotional gain of position controller & 10000 \\
$K_{d x}$ & derivative gain of position controller & 200 \\
$\omega_{x}$ & bandwidth of position control system & $100[\mathrm{rad} / \mathrm{sec}]$ \\
$K_{p g}$ & propotional gain of position controller & 2500 \\
$K_{d g}$ & derivative gain of position controller & 100 \\
$\omega_{g}$ & bandwidth of gap control system & $50[\mathrm{rad} / \mathrm{sec}]$ \\
$K_{z}$ & gain of zero-power controller & 0.002 \\
$V_{d c}$ & dc link voltage of inverter & $80[\mathrm{~V}]$ \\
$T_{s}$ & control sampling period & $50[\mu \mathrm{sec}]$ \\
$\Delta x$ & resolution of linear encoder with & \\
& quad edge evaluation method & $0.25[\mu \mathrm{m}]$ \\
$\Delta \theta$ & resolution of rotary encoder with & \\
& quad edge evaluation method & $2 \pi / 20000[\mathrm{rad}]$ \\
\hline & & \\
\hline & &
\end{tabular}

\section{B. Results}

The proposed control system is applied to spiral motor model (5)-(7). Figure 12 shows simulation results of the proposed control. The mover touches down at $t=0$ [sec], i. e., the gap displacement is set to $x_{g}=0.7[\mathrm{~mm}]$ as the initial condition. Step command $x^{c m d}=1[\mathrm{~mm}]$ for the linear position $x$ is given at $t=0.4[\mathrm{sec}]$. Figure 12(a) shows the position and gap response of the mover. In this figure, the rotation angle $\theta$ is converted to equivalent linear displacement by multiplying $\ell_{p} / 2 \pi$. We can see that the gap displacement converges to neutral position and the mover position converges to the command value without interfaring gap control. Fig. 12(b)(c) show armature current and input voltage on dq-axis.

\section{Response under Inertia Fluctuation}

Figure 13 shows simulation results when the actual mass $M$ fluctuates 10 times bigger than the nominal value $M_{n}$. Very robust response is obtained.

\section{Response under Equilibrium Fluctuation}

Figure 14-15 show simulation results under equilibrium fluctuation. The offset of equilibrium is set at $x_{g}=50[\mu \mathrm{m}]$. Figure 14 is a case with the proposed zero-power controller. The gap displacement converges to equilibrium point and zero current is realized. On the other hand, in a case without the proposed zero-power controller as shown in Fig. 15, the gap controller manages to keep $x_{g}=0$ where continuous 


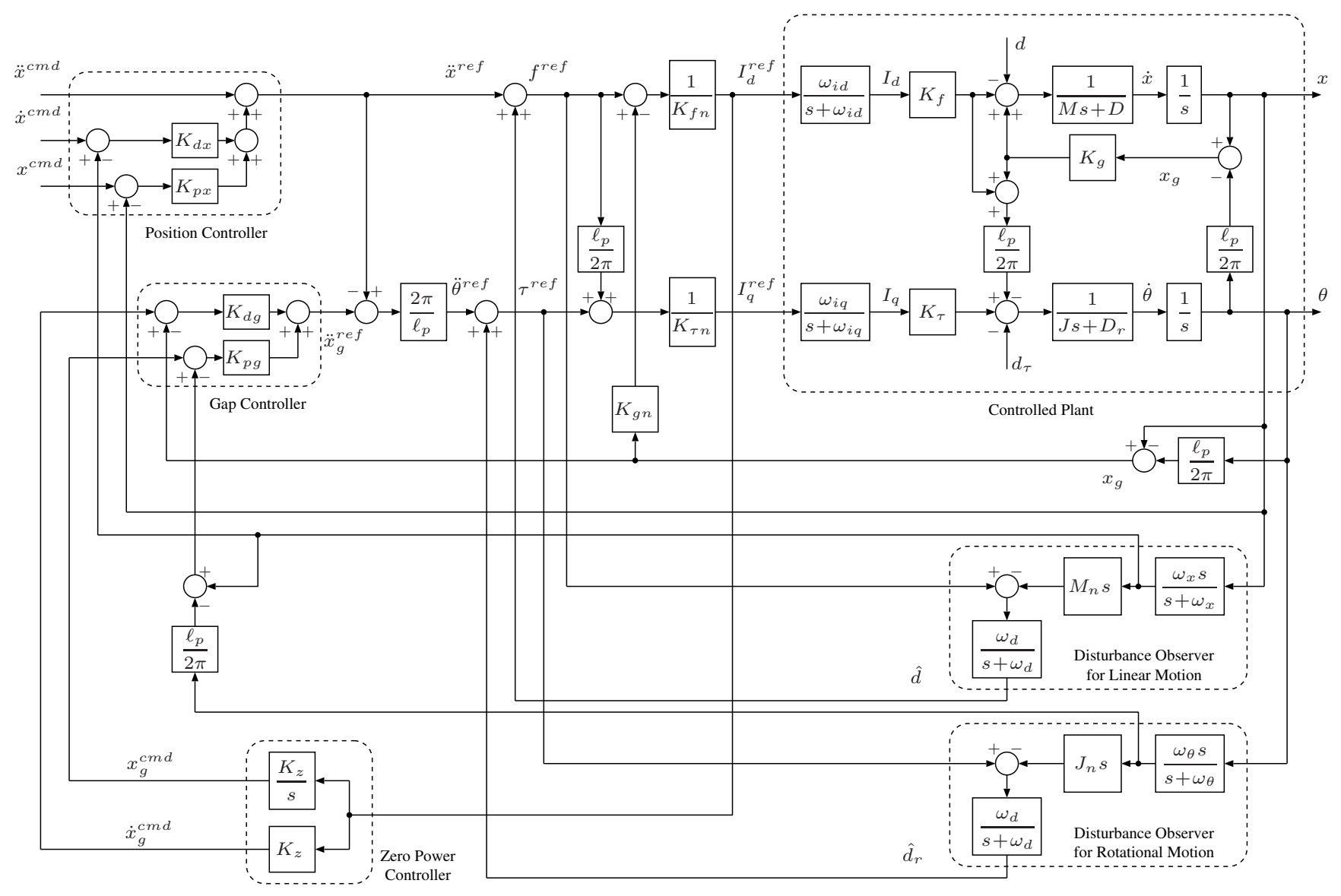

Fig. 11. Overall control system of spiral motor.

current required. Positioning accuracy of both cases are almost same. Thus, the proposed control has a advantage of energy efficiency.

\section{CONCLusion}

In this paper, modeling and control of spiral motor is proposed. The model consists of two parts; one is voltage equation that is an extension of well-known dq-axis model, and the other is motion equation that has a negative spring. The proposed controller consists of three parts; the first part is PI current controller with back EMF compensation specialized for spiral motor, the second part is disturbance observer based PD controller for linear and gap motion interacting each other, and the third part is zero-power controller for equilibrium fluctuation of gap displacement. It is comfirmed that the proposed controller achieves independent linear position and gap control simultaneously.

\section{REFERENCES}

[1] H. Asada and T. Kanade, "Design of direct-drive mechanical arms," ASME J. of Vibration, Stress, and Reliability in Design, vol. 105, no. 3, pp. 312-316, 1983.

[2] T. Murakami, F. Yu, and K. Ohnishi, "Torque sensorless control in multidegree-of-freedom manipulator," IEEE Trans. Industrial Electronics, vol. 40, no. 2, pp. 259-265, 1993.

[3] S. Katsura, Y. Matsumoto, and K. Ohnishi, "Analysis and experimental validation of force bandwidth for force control," IEEE Trans. Industrial Electronics, vol. 53, no. 3, pp. 922-928, 2006.
[4] S. Katsura, Y. Matsumoto, and K. Ohnishi, "Modeling of force sensing and validation of disturbance observer for force control," IEEE Trans. Industrial Electronics, vol. 54, no. 1, pp. 530-538, 2007.

[5] S. Katsura, K. Irie, and K. Ohishi, "Wideband force control by positionacceleration integrated disturbance observer," IEEE Trans. Industrial Electronics, vol. 55, no. 4, pp. 1699-1706, 2008.

[6] N. Hayashida, T. Yakoh, T. Murakami, and K. Ohnishi, "A sensorless force control in twin drive systems," in Proc. IEEE IECON, pp. 22312236, 2000.

[7] G. Pratt and M. Williamson, "Series elastic actuators," in Proc. IEEE IROS, pp 399-406, 1995.

[8] M. Zinn, O. Khatib, B. Roth, J. K. Salisbury, "Playing it safe," IEEE Robotics and Automation Magazine, vol. 11, no. 2, pp. 12-21, 2004.

[9] A. Bicchi and G. Tonietti, "Fast and "soft-arm" tactics," IEEE Robotics and Automation Magazine, vol. 11, no. 2, pp. 22-33, 2004.

[10] A. Bicchi, M. Bavaro, G. Boccadamo, D. De Carli, R. Filippini, G. Grioli, M. Piccigallo, G. Tonietti, R. Schiavi, and S. Sen, "Physical human-robot interaction: dependability, safety, and performance," in Proc. IEEE AMC, pp. 9-14, 2008.

[11] Y. Fujimoto, T. Kominami, and H. Hamada, "Development and analysis of a high thrust force direct-drive linear actuator," IEEE Trans. Industrial Electronics, vol. 56, no. 5, pp. 1383-1392, 2009.

[12] Y. Fujimoto, Y. Wakayama, H. Ohmori, and I. A. Smadi, "On a High-Backdrivable Direct-drive Actuator for Musculoskeletal Bipedal Robots," in proc. IEEE AMC, NF-003891, 2010.

[13] I. A. Smadi, H. Ohmori, and Y. Fujimoto, "On Independent Position/Gap Control of a Spiral Motor," in proc. IEEE AMC, NF-001899, 2010.

[14] M. Morishita, M. Akashi, and T. Azukizawa, "Zero-power Control for Maglev System of A Rigid Body Vehicle with Multi-suspended Points," IEEJ Trans. on Industry Applications, vol. 120-D, no. 4, pp. 509-519, 2000. 


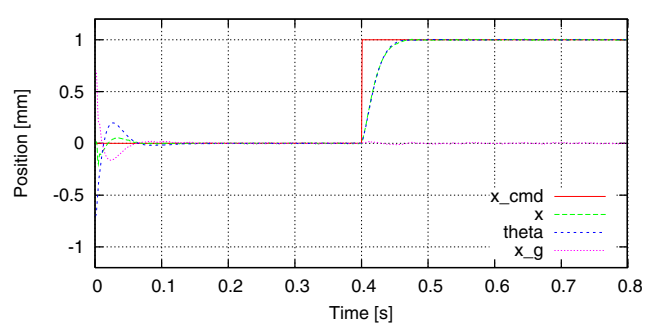

(a) Position and angle of the mover and gap displacement.

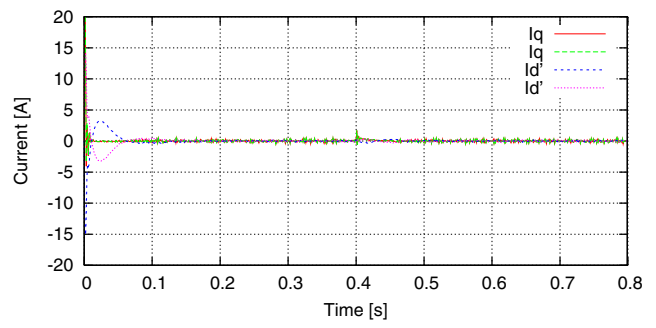

(b) Armature current.

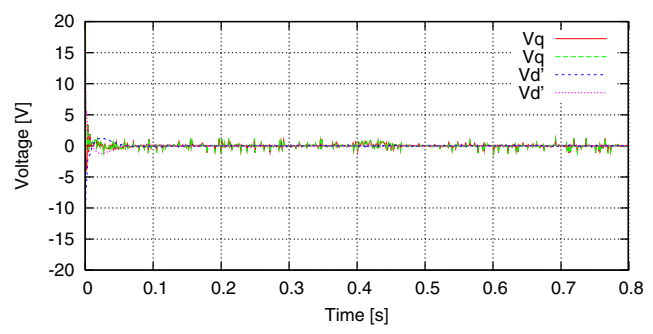

(c) Input voltage.

Fig. 12. Simulation results.

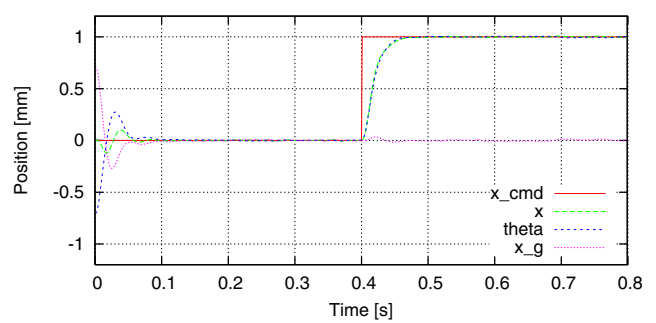

(a) Position and angle of the mover and gap displacement.

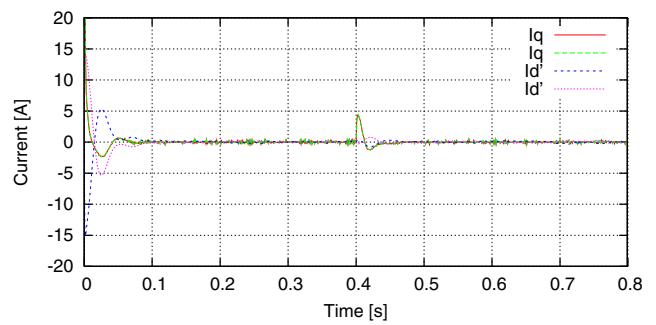

(b) Armature current.

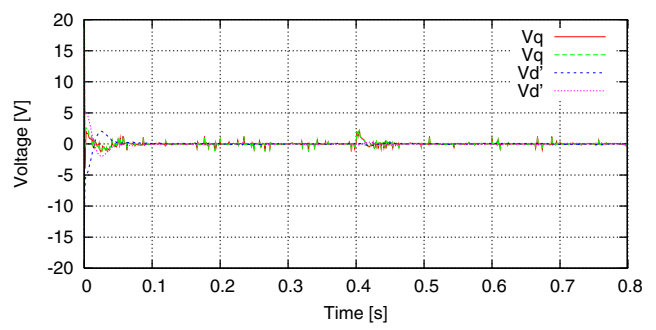

(c) Input voltage.

Fig. 13. Simulation results with mass fluctuation. $\left(M=10 M_{n}\right)$

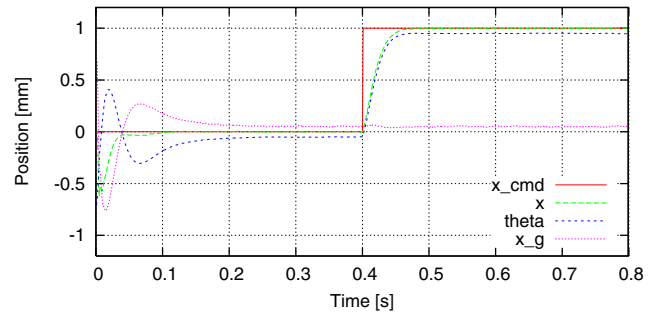

(a) Position and angle of the mover and gap displacement.

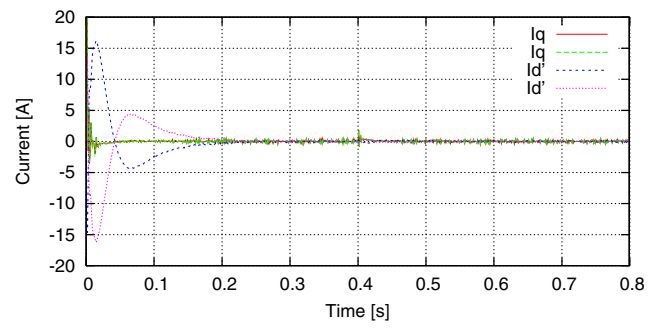

(b) Armature current.

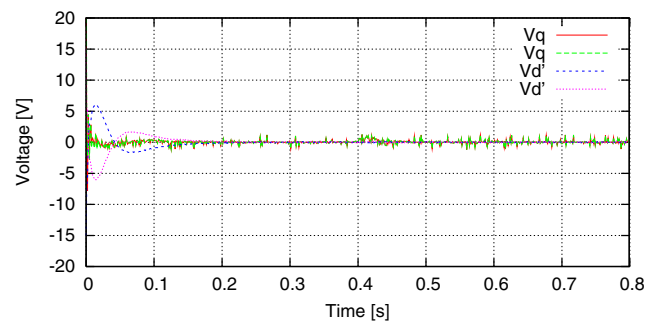

(c) Input voltage.

Fig. 14. Simulation results with equilibrium fluctuation. (offset $=50[\mu \mathrm{m}]$ )

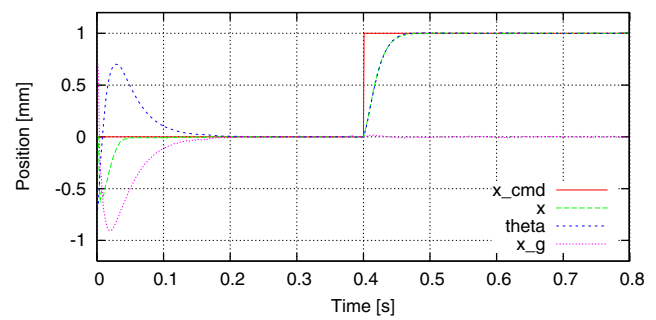

(a) Position and angle of the mover and gap displacement.

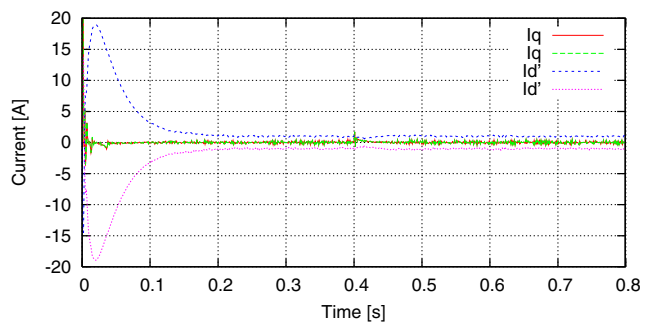

(b) Armature current.

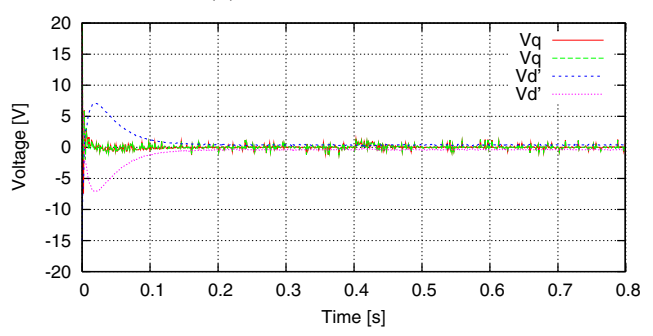

(c) Input voltage.

Fig. 15. Simulation results with equilibrium fluctuation without zero-power controller. (offset $=50[\mu \mathrm{m}])$ 